\title{
Analysis of superficial fluorescence patterns in nonmelanoma skin cancer during Photodynamic Therapy by a dosimetric model
}

\author{
I Salas-García, F. Fanjul-Vélez*, J. L. Arce-Diego* \\ Applied Optical Techniques Group, TEISA Department, University of Cantabria, Av de los Castros \\ $\mathrm{s} / \mathrm{n}, 39005$, Santander (Spain)
}

\begin{abstract}
In this work the superficial fluorescence patterns in different nonmelanoma skin cancers and their photodynamic treatment response are analysed by a fluorescence based dosimetric model. Results show differences of even more than $50 \%$ in the fluorescence patterns as photodynamic therapy progresses depending on the malignant tissue type. They demonstrate the great relevance of the biological media as an additional dosimetric factor and contribute to the development of a future customized therapy with the assistance of dosimetric tools to interpret the fluorescence images obtained during the treatment monitoring and the differential photodiagnosis.
\end{abstract}

Keywords: Photodynamic Therapy, Fluorescence, Photodiagnosis, Nonmelanoma skin cancer

\section{INTRODUCTION}

Photodynamic Therapy (PDT) consists on the administration of a nontoxic drug or dye, known as photosensitizer (PS), either systemically or topically. The administration is followed, after an incubation time, by the illumination of the lesion with visible light. This leads, in the presence of oxygen, to the generation of cytotoxic agents that produce tissue destruction [1]. During the last years it has become widely used in several clinical fields for the treatment of cancer and other types of lesions, mainly due to its minimally invasive nature and the use of non ionizing radiation. PDT has been increasingly used in dermatology as a therapeutic technique against NonMelanoma Skin Cancer (NMSC) and its precursors. The incidence of these last ones has steadily increased over the last decades due to the growth of long term exposure to ultraviolet radiation imposed by the current aesthetic tendencies [2]. However, despite the good therapeutic response, PDT dosimetry remains a challenging problem.

Despite the great effort made in recent years, the development of a complete PDT model that considers the entire complex phenomena underlying the photodynamic process has not been so far possible. Optical propagation within the tissue, PS distribution and photochemical interaction, among other effects, are crucial to evaluate the treatment progression and to estimate the treatment outcome for a given clinical context. Furthermore it would be desirable to estimate the PS fluorescence, due to the fact that it provides information relative to its degradation during treatment. Therefore it could be used as a tool for monitoring the therapy.

In this work we present a thorough analysis of PDT treatment response and fluorescence monitoring potentialities for several common NMSC tumors. The analysis assesses the relevance of the tumor type by employing a three-dimensional PDT model. Three types of skin cancer tumors, particularly nodular BCC, infiltrative BCC and squamous cell carcinoma (SCC), are considered. The whole photodynamic process is analysed, and superficial radially and depth-resolved fluorescence patterns are obtained as the treatment progresses. The specific results for each tumor type could be useful as a clinical assistance tool, both for treatment response planning and monitoring. This paper is organized as follows. Section 2 describes the dosimetric model for MAL-PDT employed. The analysis of three different types of skin cancer tumors is performed in section 3, where the main results are obtained and discussed. Finally section 4 contains the most relevant conclusions drawn from the present study.

*fanjulf@unican.es; arcedj@unican.es; phone +34 94220 67 30; fax +34 9422018 73; www.teisa.unican.es/toa 


\section{DOSIMETRIC MODEL}

The model employed in this work considers the main phenomena in the above mentioned photodynamic process. A brief description of the approaches used to model each of them is given below.

\subsection{Topical photosensitizer precursor distribution and accumulation of photoactive agent}

Fick's law was employed to obtain the inhomogeneous spatial-temporal distribution of the topical PS precursor in the target tissue during the 3 hours incubation period imposed by Metvix ${ }^{\circledR}$ protocol. The permeability through the damaged stratum corneum in abnormal tissue $K$ and the diffusion coefficient within the tissue $D$ were taken into account as it is expressed in Eq. (1) [3]. In this equation $\tau$ is the relaxation time of the precursor as a consequence of the generation of the photosensitizer and other processes such as the lymphatic flow and the blood perfusion, and $M_{0}$ is the initial concentration of precursor applied in the tumor surface. The initial precursor concentration applied to the tumor surface was obtained from the indications given by the protocol specifications related to the Metvix ${ }^{\circledR}$ cream administration, assuming a lesion with a circular superficial extension, the precursor density and its molecular mass. The endogenously produced photoactive compound $S_{0}$ is proportional to the instantaneous value of the precursor concentration when the photosensitizer relaxation time $\tau_{p}$ is short compared to its precursor diffusion time $t$ and can be obtained by Eq. (2). Where $\varepsilon_{p}$ is the conversion process yield and $\tau_{a \rightarrow p}$ the relaxation time of the photosensitizer precursor due to the generation of the photoactive compound.

$$
\begin{gathered}
M(t)=M_{o} \int_{0}^{t}\left(\frac{K}{\sqrt{D \pi t^{\prime}}} e^{-\frac{z^{2}}{4 D t^{\prime}}}-\frac{K^{2}}{D} e^{\frac{K}{D} z} e^{\frac{K^{2}}{D} t^{\prime}} \operatorname{erfc}\left(\frac{K}{\sqrt{D}} \sqrt{t^{\prime}}+\frac{z}{2 \sqrt{D t^{\prime}}}\right)\right) e^{-\frac{t^{\prime}}{\tau}} d t^{\prime} \\
S_{0}(t)=\varepsilon_{p} \frac{\tau_{p}}{\tau_{a \rightarrow p}} M(t)
\end{gathered}
$$

\subsection{Photochemical interaction}

The photochemical pathways during a type II PDT process were modeled by a differential equations system (3-8), in order to obtain the temporal evolution of the molecular components involved in the photochemical interaction [4, 5]. In these equations $S_{0}$ is the photosensitizer in ground state whose initial value is that previously obtained after the incubation period. $S_{1}$ stands for the photosensitizer in singlet excited state and $T$ in triplet excited state. ${ }^{3} O_{2}$ is the oxygen in ground state, ${ }^{1} \mathrm{O}_{2}$ is the singlet oxygen, $\mathrm{R}$ are the intracellular ${ }^{1} \mathrm{O}_{2}$ receptors and $\mathrm{C}$ the oxygen scavengers; $\tau 1$ is the relaxation time from state $S_{1}$ to $S_{0} ; \tau 3$ is the relaxation time from state $T$ to $S_{0} ; \tau 0$ is the relaxation time from state ${ }^{1} O_{2}$ to ${ }^{3} O_{2} ; \eta_{10}$ is the quantum yield of the $S_{1}$ to $S_{0}$ transition; $\eta_{13}$ is the quantum yield of the $S_{1}$ to $T$ transition; $\eta_{30}$ is the quantum yield of $T$ transition to $S_{0} ; \eta_{0}$ is the quantum yield of ${ }^{1} O_{2}$ transition to ${ }^{3} O_{2} ; \alpha s$ is the efficiency factor for energy transfer from $\mathrm{T}$ to ${ }^{3} \mathrm{O}_{2} ; \mathrm{kpb}$ stands for the biomolecular photobleaching rate; $k c x$ is the biomolecular cytotoxicity rate; $k s c$ is the rate of reaction with various oxygen scavengers; $v$ is the light speed in tissue; $\rho$ is the photon density; $\sigma_{p s a}$ is the absorption cross section of molecules; $P$ is the rate of oxygen diffusion and perfusion; and $U$ is the cell damage repair rate. The photosensitizer in ground state, whose initial value is that previously obtained after the incubation period, is among the required initial conditions to solve it. The stiff differential equations system was solved by means of a differential equation solver within the Matlab ${ }^{\circledR}$ platform. 


$$
\begin{aligned}
& \frac{d\left[S_{0}\right]}{d t}=-v \rho \sigma_{p s a}\left[S_{0}\right]-k p b\left[{ }^{1} O_{2}\right]\left[S_{0}\right]+\frac{\eta_{10}}{\tau 1}\left[S_{1}\right]+\frac{\eta_{30}}{\tau 3}[T]+\frac{\alpha s}{\tau 3}[T]\left[{ }^{3} O_{2}\right] \\
& \frac{d\left[S_{1}\right]}{d t}-\frac{1}{\tau 1}\left[S_{1}\right]+v \rho \sigma_{p s a}\left[S_{0}\right](4) \\
& \frac{d[T]}{d t}=-\frac{\eta_{30}}{\tau 3}[T]-\frac{\alpha s}{\tau 3}[T]\left[{ }^{3} O_{2}\right]+\frac{\eta_{13}}{\tau 1}\left[S_{1}\right](5) \\
& \frac{d\left[^{3} O_{2}\right]}{d t}=-\frac{\alpha s}{\tau 3}[T]\left[{ }^{3} O_{2}\right]+\frac{\eta_{0}}{\tau 0}\left[{ }^{1} O_{2}\right]+P(6) \\
& \frac{d\left[{ }^{1} O_{2}\right]}{d t}=-k p b\left[S_{0}\right]\left[{ }^{1} O_{2}\right]-k c x[R]\left[{ }^{1} O_{2}\right]-k s c[C]_{i}\left[{ }^{1} O_{2}\right]-\frac{\eta_{0}}{\tau_{0}}\left[{ }^{1} O_{2}\right]+\frac{\alpha s}{\tau 3}[T]\left[{ }^{3} O_{2}\right](7) \\
& \frac{d[R]}{d t}=-k c x\left[{ }^{1} O_{2}\right][R]+U(8)
\end{aligned}
$$

\subsection{Propagation of the optical excitation and superficial fluorescence patterns}

Optical modeling in a three-dimensional tissue can be addressed through the Radiation Transport Theory (RTT), assuming multiple scattering, and neglecting therefore polarization or interference effects. Without sources inside the tissue and in a steady-state situation, the differential RTT can be expressed as in Eq. (9), where the basic parameter of light is the specific intensity $I(r, \hat{s})$ or light power per unit area per unit solid angle. The radiation is expected to be at point $\vec{r}$, and to follow the direction $\hat{s}$. The scattering events are treated according to the scattering phase function $p\left(\hat{s} \cdot \hat{s}^{\prime}\right)$. Optical radiation comes from direction $\hat{s}^{\prime}$ and is redirected to $\hat{s}$.

$$
\hat{s} \cdot \bar{\nabla} I(r, \hat{s})=-\left(\mu_{a}+\mu_{s}\right) I(r, \hat{s})+\frac{\mu_{s}}{4 \pi} \int_{4 \pi} p\left(\hat{s} \cdot \hat{s}^{\prime}\right) I\left(r, \hat{s}^{\prime}\right) d \Omega^{\prime}(9)
$$

The Monte Carlo method by Wang and Jacques [6] was employed to solve it. This implementation is multi-layered, with their borders always perpendicular to the laser beam. This assumption is adequate for a stratified media as the skin. The approach employs the optical parameters of each tissue layer at the PDT treatment wavelength $(635 \mathrm{~nm})$, mainly the index of refraction $n$, the absorption coefficient $\mu_{a}$, the scattering coefficient $\mu_{s}$ and the anisotropy of scattering $g$ as well as its dimensions are required.

The time-resolved three dimensional PS fluorescence emission at $705 \mathrm{~nm}$ was obtained taking into account the excitation photon density absorbed by the PS molecules and the fluorescence quantum yield $\eta_{10}$ [7]. The temporal evolution of the fluorescence power density generated by the PS molecules as the treatment progresses can be expressed as in Eq. (10). In this equation $v$ is the light speed in tissue, $\rho$ is the photon density at a point and $\sigma_{p s a}$ is the absorption cross-section of the PS molecules. $E_{\text {photon } n_{\text {em }}}=h \cdot c / \lambda_{e m}$ is the photon energy at the fluorescence emission wavelength $\lambda_{e m}, h$ is the Planck's constant and $c$ is the speed of light in vacuum.

$$
P_{f}(r, z, t)=v \cdot \rho \cdot \sigma_{p s a} \cdot\left[S_{0}\right] \cdot \eta_{10} \cdot E_{\text {photon } \text { Rem }_{\text {em }}}(1
$$

A time-dependent spatially non uniform fluorophore distribution produces time-resolved fluorescence emission sources at every spatial position of the tissue sample during the treatment. These sources were considered to obtain the fluorescence escape at the tumor surface. Superficial fluorescence due to the PS molecules was calculated by means of another Monte Carlo approach [8]. The method was modified to consider local fluorescence sources that are dynamically updated over time and space. Thus, for a particular treatment time, the total escaping fluorescence $J_{f}$ at a certain superficial radial position $r$ can be obtained by the accumulation of the escaping flux produced by each of the fluorescent power sources, as it is expressed in Eq. (11). In this equation $r_{s}$ and $z_{s}$ are the coordinates of the 
fluorescence source, $\Delta V\left(r_{s}, z_{s}\right)$ is the incremental volume associated to the source position, and $T\left(r_{s}, z_{s}, r\right)$ is the fluorescence transfer function from the source to the tissue surface [9].

$J_{f}(r, t)=\sum_{r_{s}} \sum_{z_{s}} P_{f}\left(r_{s}, z_{s}, t\right) \Delta V\left(r_{s}, z_{s}\right) T\left(r_{s}, z_{s}, r\right)(11)$

\section{RESULTS AND DISCUSSION}

The MAL-PDT model outlined in the previous section was applied to three different types of NMSC: infiltrative BCC, nodular BCC and SCC. The lesion geometry was considered to have a superficial radius of $1 \mathrm{~cm}$ and $3 \mathrm{~mm}$ depth for all of them. Their optical properties at the treatment and fluorescence emission wavelengths were extracted from [10] (see table 1), and the same optical source was applied in all the cases. A top hat laser beam with $0.3 \mathrm{~cm}$ radius perpendicular to the target was used to deliver an irradiance of $100 \mathrm{~mW} / \mathrm{cm}^{2}$. The total treatment time was set to 10 minutes. MAL initial concentration applied on the surface of the lesion was calculated taking into account the Metvix ${ }^{\circledR}$ clinical protocol specifications. This concentration specifies that Metvix ${ }^{\circledR}$ cream is applied in a $1 \mathrm{~mm}$ thick layer on the affected area covering an extra $5 \mathrm{~mm}$ of healthy skin around the damaged area during an incubation period of $3 \mathrm{~h}$ before the irradiation. Therefore taking into account a MAL density of $160 \mathrm{mg}$ per gram of Metvix ${ }^{\circledR}$ cream and the molecular mass of MAL, the initial concentration of the photosensitizer precursor on the pathology surface employed was $4.5 \cdot 10^{20} \mathrm{~cm}^{-3}$. Common values for the diffusion coefficient through the epidermis and dermis, $0.69 \cdot 10^{-10} \mathrm{~m}^{2} / \mathrm{s}$ and for the permeability of the stratum corneum, $10^{-6} \mathrm{~m} / \mathrm{s}$ were adopted. More detailed information related to the values assigned to the parameters involved in the photochemical interaction can be found in our previous work [11].

Table 1. Optical properties of tissues at the treatment and fluorescence emission wavelengths [10].

\begin{tabular}{|c|c|c|c|c|}
\hline \multirow{2}{*}{ Type of tissue } & \multicolumn{2}{|c|}{ Excitation wavelength $(635 \mathrm{~nm})$} & \multicolumn{2}{c|}{ Emission wavelength $(705 \mathrm{~nm})$} \\
\cline { 2 - 5 } & $\mu_{\mathrm{a}}\left(\mathrm{cm}^{-1}\right)$ & $\mu_{\mathrm{s}}\left(\mathrm{cm}^{-1}\right)$ & $\mu_{\mathrm{a}}\left(\mathrm{cm}^{-1}\right)$ & $\mu_{\mathrm{s}}\left(\mathrm{cm}^{-1}\right)$ \\
\hline Infiltrative BCC & 1.5 & 142.85 & 1.5 & 100 \\
\hline Nodular BCC & 1.5 & 104.76 & 0.8 & 90 \\
\hline SCC & 2 & 95.238 & 1 & 85 \\
\hline
\end{tabular}

Results shown in Fig. 3 reveal differences in the radially-resolved surface fluorescence patterns obtained as the treatment progresses depending on the malignant tissue type. Although the spatiotemporal trend was quite similar for the three types of skin tumor (nodular BCC, infiltrative BCC and SCC) under the same treatment conditions, fluorescence irradiance varies for each lesion at every treatment time (Figs. 3a), b), c) and d)). The statistical quantification of these differences within the optical source radius reveals a mean increase (over $50 \%$ ) between the nodular BCC and the infiltrative BCC during all the treatment time (50.39\% at $60 \mathrm{~s}, 58.22 \%$ at $300 \mathrm{~s}$ and $56.51 \%$ at $600 \mathrm{~s})$. This difference is slightly reduced between the SCC and the infiltrative BCC to $35.30 \%, 40.62 \%$ and $39.46 \%$ at $60 \mathrm{~s}, 300 \mathrm{~s}$ and $600 \mathrm{~s}$, respectively. The discrimination among diseases is considerably reduced when we compare the nodular BCC and the SCC. In this last case, the difference is reduced to $11.18 \%, 12.53 \%$ and $12.25 \%$. This analysis is of great help when attempting to monitor the evolution of a personalized treatment. The planning must take into account the particular tumor type of the patient, which significantly modifies the fluorescence pattern as shown in the previous results. The fluorescence pattern has a direct relationship with the PS degradation during the photochemical interaction, and consequently with the cytotoxic agent generation. As it can be appreciated in Fig. 3a), differences are even present at only $1 \cdot 10^{-8} \mathrm{~s}$ from the beginning of the irradiation period, when the cytotoxic agent has not yet been produced. Therefore the predictive model used in this work could also be useful as a supporting tool for diagnostic (not treatment-oriented) purposes, in what is known as photodynamic diagnosis. The statistically significant differences in the superficial 
fluorescence emission, as well as the lack of overlap between the standard deviations represented by error bars in Fig. 3, support its viability as diagnostic tool.
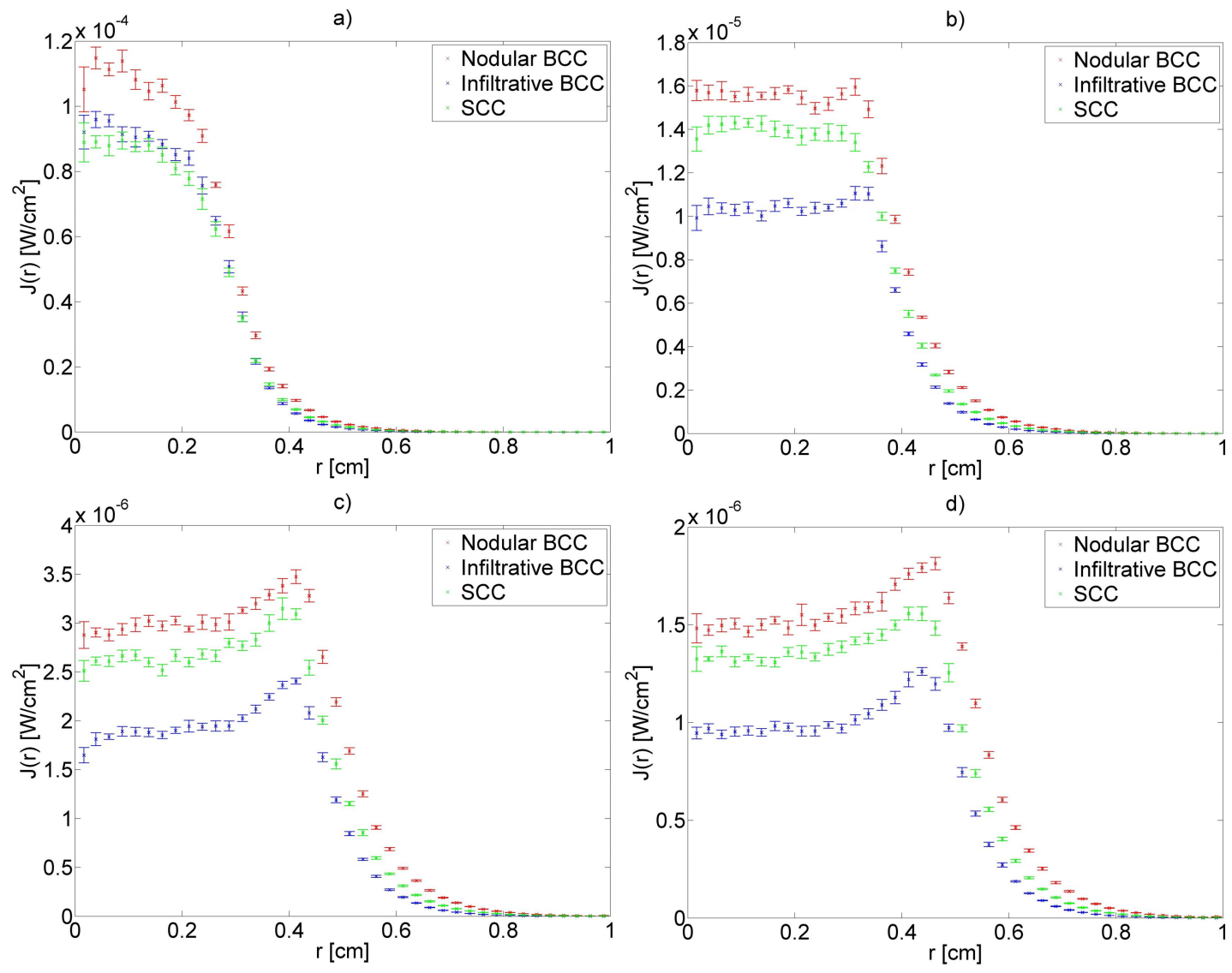

Figure 3. Surface radially-resolved fluorescence of nodular BCC, infiltrative BCC and SCC malignant tissues (W/ $\mathrm{cm}^{2}$ ) vs. the radial component at a) $1 \cdot 10^{-8} \mathrm{~s}$, b) $60 \mathrm{~s}$, c) $300 \mathrm{~s}$ and d) $600 \mathrm{~s}$.

\section{CONCLUSIONS}

Three different types of NMSC (nodular BCC, infiltrative BCC and SCC) have been analysed by a predictive threedimensional time-resolved photochemical and radially-resolved fluorescence model for MAL-PDT. Results reveal differences of even more than $50 \%$ in the superficial fluorescence patterns obtained as the treatment progresses, depending on the type of malignant tissue. Surface fluorescence is related with the cytotoxic effect of the PDT treatment. As a consequence the tumor and lesion type of the patient must be considered in the predictive models for appropriate treatment planning and monitoring that avoids tumor recurrence. The results could be also useful for differential photodynamic diagnosis.

\section{ACKNOWLEDGEMENTS}

This work has been partially supported by the project MAT2012-38664-C02-01 of the Spanish Ministery of Economy and Competitiveness and by San Cándido Foundation. The authors thank Marqués de Valdecilla University Hospital for providing information about actual medical praxis. 


\section{REFERENCES}

[1] Hamblin, M. R. and Mróz, P., "Advances in Photodynamic Therapy: Basic, Translational and Clinical," Engineering in medicine \& Biology, (2008).

[2] Choudhary, S., Tang, J., Elsaie, M. L. and Nouri, K., "Lasers in the Treatment of Nonmelanoma Skin Cancer," Dermatol. Surg. 37, 409-425 (2011).

[3] Svaasand, L. O., Wyss, P., Wyss, M. T., Tadir, Y., Tromberg, B. J. and Berns, M. W., "Dosimetry model for photodynamic therapy with topically administered photosensitizers," Lasers in Surgery and Medicine 18, 139149 (1996).

[4] Foster, T. H., Murant, R. S., Bryant, R. G., Knox, R. S., Gibson, S. L. and Hilf, R., "Oxygen consumption and diffusion effects in PDT," Radiation Research 126(3), 296-303 (1991).

[5] Hu, X. H., Feng, Y., Lu, J. Q., Allison, R. R., Cuenca, R. E., Downie, G. H. and Sibata, C. H., "Modeling of a type II photofrin-mediated PDT process in a heterogeneous tissue phantom," Photochemistry and Photobiology 81, 1460-1468 (2005).

[6] Wang, L., Jacques, S. L. and Zheng, L., "MCML - Monte Carlo modeling of light transport in multi-layered tissues," Comput. Meth. Programs Biomed. 47, 131-146 (1995).

[7] Salas-García, I., Fanjul-Vélez, F. and Arce-Diego, J. L., "Spatial photosensitizer fluorescence emission predictive analysis for photodynamic therapy monitoring applied to a skin disease," Opt. Commun. 285, 15811588 (2012).

[8] Rogue, B. W. and Mycek, M. A., [Handbook of Biomedical Fluorescence], CRC Press, 61-107 (2003).

[9] Salas-García, I., Fanjul-Vélez, F. and Arce-Diego, J. L., "Superficial radially-resolved fluorescence and threedimensional photochemical time-dependent model for Photodynamic Therapy,” Opt. Lett. 39, 1845-1848 (2014).

[10] Salomatina, E., Jiang, B., Novak, J. and Yaroslavsky, A. N., "Optical properties of normal and cancerous human skin in the visible and near-infrared spectral range," J. Biomed. Opt. 11, 0640261-640269 (2006).

[11] Salas-García, I., Fanjul-Vélez, F. and Arce-Diego, J. L., "Photosensitizer absorption coefficient modeling and necrosis prediction during photodynamic therapy," J. Photochem. Photobiol. B-Biol. 114, 79-86 (2012). 